Stimulated forward Raman scattering in large scale-length laser-produced plasmas

C. Niemann, R. L. Berger, L. Divol, R. K. Kirkwood, J. D. Moody, C. M. Sorce, S. H. Glenzer

August 26, 2011

Journal of Instrumentation (JIST) 
This document was prepared as an account of work sponsored by an agency of the United States government. Neither the United States government nor Lawrence Livermore National Security, LLC, nor any of their employees makes any warranty, expressed or implied, or assumes any legal liability or responsibility for the accuracy, completeness, or usefulness of any information, apparatus, product, or process disclosed, or represents that its use would not infringe privately owned rights. Reference herein to any specific commercial product, process, or service by trade name, trademark, manufacturer, or otherwise does not necessarily constitute or imply its endorsement, recommendation, or favoring by the United States government or Lawrence Livermore National Security, LLC. The views and opinions of authors expressed herein do not necessarily state or reflect those of the United States government or Lawrence Livermore National Security, LLC, and shall not be used for advertising or product endorsement purposes. 


\title{
Stimulated forward Raman scattering in large scale-length laser-produced plasmas
}

\author{
C. Niemann ${ }^{1}$, R.L. Berger ${ }^{2}$, L. Divol ${ }^{2}$, R.K. Kirkwood ${ }^{2}$, J.D. \\ Moody $^{2}$, C.M. Sorce ${ }^{2}$, and S.H. Glenzer ${ }^{2}$ \\ ${ }^{1}$ University of California Los Angeles, 1040 Veteran Avenue, CA 90095, USA \\ ${ }^{2}$ Lawrence Livermore National Laboratory, 7000 East Avenue, Livermore, CA 94550, \\ USA \\ E-mail: cniemann@ucla.edu
}

\begin{abstract}
A forward stimulated Raman scattering (FSRS) diagnostic was developed for the 60 beam Omega laser facility to investigate the propagation of an intense $\left(\sim 8 \times 10^{14} \mathrm{~W} / \mathrm{cm}^{2}\right)$, frequency doubled Nd:glass laser beam ( $\left.\leq 360 \mathrm{~J}, 527 \mathrm{~nm}, 1 \mathrm{~ns}\right)$ through a mm-scale laser-produced plasma. Forward scattered light was measured with spectral, and temporal resolution using a streaked spectrometer and an absolutely calibrated photo-multiplier. We present a detailed description of the instrument, the calibration methods, as well as the first forward Raman scattering measurements from hot $(\sim 2 \mathrm{keV})$, dense $\left(5.5 \times 10^{20} \mathrm{~cm}^{-3}\right)$ laser-produced plasmas. These results are of interest to laser-driven inertial fusion at the National Ignition Facility where larger plasma scales could potentially lead to higher FSRS gains. In addition, simultaneous measurements of stimulated forward and backward scattered light present an unambiguous method for determining plasma density and temperature.
\end{abstract}




\section{Introduction}

Understanding laser-light scattering in large scale-length laser produced plasmas is important for indirect drive inertial confinement fusion [1]. Stimulated forward Raman scattering (FSRS) produces energetic electrons in excess of $1 \mathrm{MeV}$, which can preheat the fuel capsule and potentially degrade laser-fusion target gain. Forward Raman scattering is the parametric decay of a laser light wave into a scattered electromagnetic wave and an electron plasma wave (EPW). Since the process obeys the wavenumber- and frequencymatching conditions $k_{0}=k_{s}+k_{\text {epw }}$ and $\omega_{0}=\omega_{s}+\omega_{\text {epw }}$, and all k-vectors point in the same direction, the Langmuir wave-vector $k_{e p w}$ is small. Its phase velocity $\omega_{e p w} / k_{e p w}$ is large (comparable to the speed of light for ignition conditions) and there are essentially no electrons to damp the wave.

The gain of Backward Stimulated Raman scattering (SRS), which is the primary scattering mechanism in laser-fusion hohlraums [2], is very sensitive to the electron temperature and density because its growth rate is limited by Landau damping. In contrast, the gain of FSRS is practically independent of the plasma temperature but depends more critically on density gradients, as the weak wave damping means the resonance width is very narrow. Previous FSRS measurements in laser-produced plasmas were performed at the NOVA laser using thin foil targets to minimize density gradients [3]. In those earlier experiments the total level of FSRS was several orders of magnitude below the level of backward SRS and was mostly observed at densities much higher than current target designs for laser-fusion. Here we present results from a new diagnostic developed for the 60 beam Omega laser facility [4] and forward Raman scattering measurements from laser-heated gasbag targets which have larger, more homogeneous plasmas than the thin foil targets. The results reported here use fusion-relevant laser intensities and plasma densities along with modern beam smoothing techniques [5]. Significantly higher FSRS levels are found in these plasmas, which indicates that forward scattering may also be important in laser-fusion experiments on the National Ignition Facility (NIF).

\section{Forward Raman scattering diagnostics}

The forward Raman scattering diagnostic described here is an upgrade to a transmitted laser beam diagnostic for a frequency doubled $(2 \omega, 527 \mathrm{~nm})$ interaction beam [6], presented earlier [7]. A 3 inch diameter spherical fused silica bare-surface mirror is positioned inside the target chamber, $23 \mathrm{~cm}$ behind the laser-target using a robotic arm (Fig. 1a). The mirror reflects $4 \%$ of the transmitted light over twice the initial $\mathrm{f} / 6.7$ cone of the interaction beam through a port window to the detector assembly outside the vacuum chamber. The uncoated concave front side of the mirror $(\mathrm{R}=37 \mathrm{~cm})$ reflects the divergent beam behind the target as a convergent beam towards the chamber port,

producing a focus inside the vacuum chamber and a divergent $\mathrm{f} / 13$ beam with a diameter of $3.8 \mathrm{~cm}$ at the port window. The back-side of the mirror has a high-intensity laser 
anti-reflex (AR) coating for $527 \mathrm{~nm}$ light to reduce ghosts.

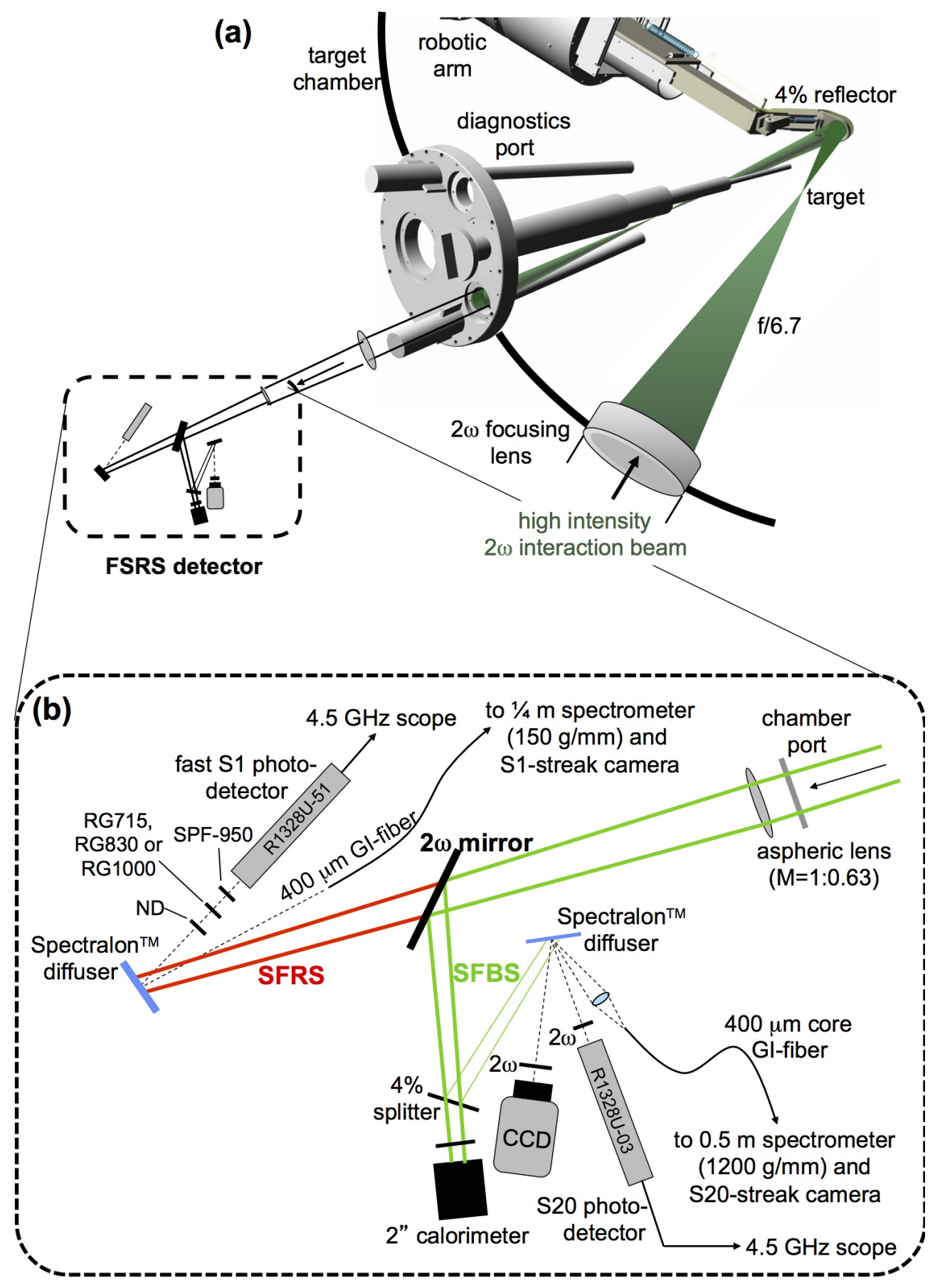

Figure 1. (a) Transmitted and forward Raman scattered light is collected with a highdamage threshold mirror behind the target and relay imaged to a detector assembly outside the vacuum chamber. (b) Upshifted FSRS light and transmitted light at the laser wavelength are separated using a dichroic mirror and measured with absolutely calibrated photo-multiplier tubes and fiber-coupled streaked spectrometers.

An aspheric lens (f/7.9) outside the chamber relay-images the collection mirror plane onto Lambertian diffuser plates (Spectralon ${ }^{\mathrm{TM}}$ ) with a high damage threshold 
$\left(>4 \mathrm{~J} / \mathrm{cm}^{2}\right)$ and flat spectral response. Up-shifted light due to stimulated forward Raman scattering (around $800 \mathrm{~nm}$ depending on plasma density and temperature) is separated from transmitted light at the probe beam wavelength $(527 \mathrm{~nm})$ with a dichroic mirror behind the lens (Fig. 1b). Both the diffuser plate for $2 \omega$ light and FSRS light are viewed with absolutely calibrated, filtered photo-multiplier tubes and fiber-coupled spectrometers.

SFRS light from the diffuser is collected by a $16 \mathrm{~m}$ long $400 \mu \mathrm{m}$ graded-index fiber with flat spectral response in the range of interest (Mitsubishi SGLH400E, $5 \mathrm{~dB} / \mathrm{km}$ attenuation between 0.5 and $1 \mu \mathrm{m}$ ) and sent to a SPEX $1 / 4 \mathrm{~m}$ spectrometer and streak camera with S1 cathode. A separate optical fiber from a backward scattering diagnostics [6] was coupled to the same spectrometer to obtain a simultaneous measurement of both forward and backward scattered light. The two fiber lengths were adjusted to introduce a 2.5 ns relative delay between the FSRS and the SRS signals. The spectral and temporal resolution of the system using a low dispersion 150 lines/mm grating was $1 \mathrm{~nm}$ and 50 ps, respectively.

Large area photo-multiplier tubes coupled to a $4.5 \mathrm{GHz}$ oscilloscope were used to obtain absolutely calibrated measurements of the transmitted light and the forward scattered light (Hamamatsu R-1328U-51 with S1 cathode for FSRS and R1328U-03 with S20 cathode for $2 \omega$ ). A line filter with $5 \mathrm{~nm}$ bandwidth (fwhm) was used for the $2 \omega$ detector and a combination of short-pass (SPF950 to block out unconverted laser light at $1053 \mathrm{~nm}$ ) and long-pass filters (RG715, RG830 or RG1000 to block out transmitted light at $527 \mathrm{~nm}$ ) were used for the forward scattered light detector.

We chose this alignment insensitive design with diffuser plates but reduced light collection efficiency since no alignment beam at the upshifted wavelength (around 800 $\mathrm{nm}$ ) was available. Both the detectors for unshifted and upshifted light were aligned using a continuous wave laser beam at $527 \mathrm{~nm}$ and by replacing the dichroic splitter with an uncoated glass-blank of the same optical thickness.

\section{Calibration procedure}

Absolute measurements of FSRS light levels and transmitted $2 \omega$ levels were performed with two large aperture photo-multiplier tubes which were calibrated off-line with a pulsed low-energy laser beam (Continuum MiniLite ${ }^{\mathrm{TM}}$ ). The calibration beam (up to $10 \mathrm{~mJ}$ in $5 \mathrm{~ns}$ at $532 \mathrm{~nm}$ ) was fired at the diffuser plate and the reflected signal was measured with the photo-tube, while the absolute beam energy was monitored with a pyroelectric detector (Molectron J8LP). The incidence and viewing angle, as well as the beam diameter on the plate, and distance of the photo-tube were chosen such as to reproduce the actual experimental setup. We integrate the photo-tube signal over the entire $5 \mathrm{~ns}$ pulse to determine an equivalent energy in $\mathrm{V} \cdot \mathrm{ns}$. The detector response at these conditions was measured to be $(6.1 \pm 0.2) \times 10^{-5} \mathrm{~J} /(\mathrm{V} \cdot \mathrm{ns})$ at $532 \mathrm{~nm}$. The signal dependence on angles and distances was also investigated and the measurement was repeated several times using different setups to determine a combined error bar of $3 \%$. 
Since both the detectors for unshifted light $(527 \mathrm{~nm})$ and upshifted FSRS light $(\sim$ $800 \mathrm{~nm}$ ) were absolutely calibrated using the same pulsed laser at $532 \mathrm{~nm}$, we used the spectral response curves for the photo-tubes provided by the manufacturer to translate this calibration to the $800 \mathrm{~nm}$ range [8]. To obtain an accurate calibration of the forward scattering detector in the optical system the spectral response of all optical components (window, dichroic splitter, mirrors, filters etc.) was measured separately between 500 $\mathrm{nm}$ and $1000 \mathrm{~nm}$ using a solid-state plasma light source with known spectral output and a grating spectrometer (Fig. 2).

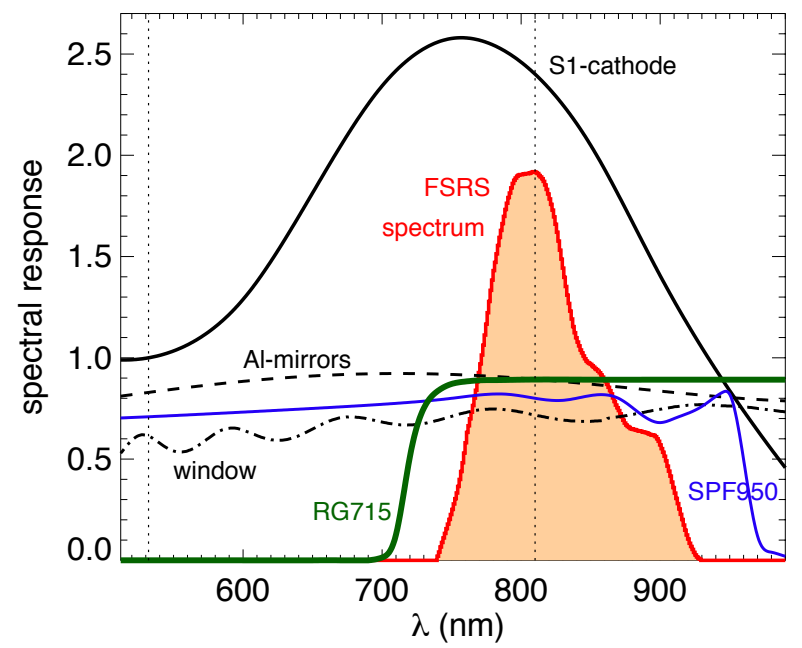

Figure 2. Measured spectral response of key optical components that contribute to the absolute sensitivity of the photo-multiplier tube. The measured FSRS spectrum considering the spectral response of the spectrometer is plotted in arbitrary units for comparison (solid filled red line, see section 4 for details). The S1-cathode of the phototube shows the largest variation in sensitivity with wavelength, while the aluminum coated mirrors, the vacuum window, and the long- and short-pass filters are mostly flat over a broad spectral range. The graph shows absolute transmission or reflectivity for the optical components while the cathode sensitivity was normalized to $532 \mathrm{~nm}$.

Figure 3(a) shows the overall system response $R(\lambda)$ for the three long-pulse filter combinations used in the experiment (i.e. RG715, RG830 and RG830 + RG1000). We note that $R(\lambda)$ with the RG715 filter peaks above the laser-line calibration at $532 \mathrm{~nm}$, since the sensitivity of the S1 cathode increases towards $750 \mathrm{~nm}$. Measuring the photodetector signal with different filters for similar laser-plasma conditions and FSRS levels can thus give a rough estimate of the spectral distribution of the forward scattered light and can be used to validate the streaked spectrometer data. The actual photo-tube calibration-factor for a given filter $R_{\text {filter }}$ was calculated from the known spectrum $\mathrm{S}(\lambda)$ and the overall system response $\mathrm{R}(\lambda)$ using

$$
R_{\text {filter }}=6.1 x 10^{-5} \mathrm{~J} /(\mathrm{Vns}) \cdot \sum_{\lambda} S(\lambda) / \sum_{\lambda} R(\lambda) S(\lambda)
$$

where $6.1 \times 10^{-5} \mathrm{~J} /(\mathrm{Vns})$ is the detector sensitivity for a discrete spectrum at $532 \mathrm{~nm}$ as 
determined in the pulsed laser calibration. The actual sensitivity of the photo-detector is therefore $\mathrm{R}_{750}=7.1 \times 10^{-5} \mathrm{~J} /(\mathrm{V} \mathrm{ns})$ with the $\mathrm{RG} 715$ filter, $\mathrm{R}_{830}=2.4 \times 10^{-4} \mathrm{~J} /(\mathrm{V} \mathrm{ns})$ with the RG830 filter, and $R_{850+1000}=1.8 \times 10^{-2} \mathrm{~J} /(\mathrm{V}$ ns $)$ with both the RG830 and the RG1000 installed (Fig. 3(b)).

(a)

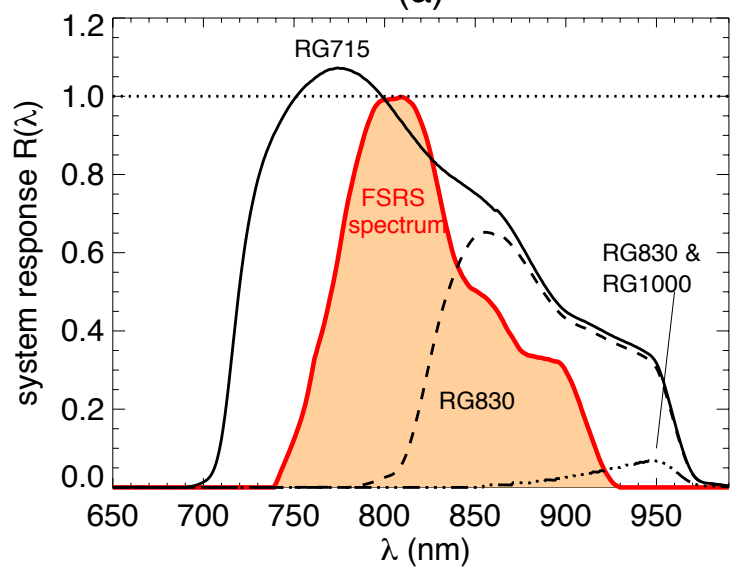

(b)

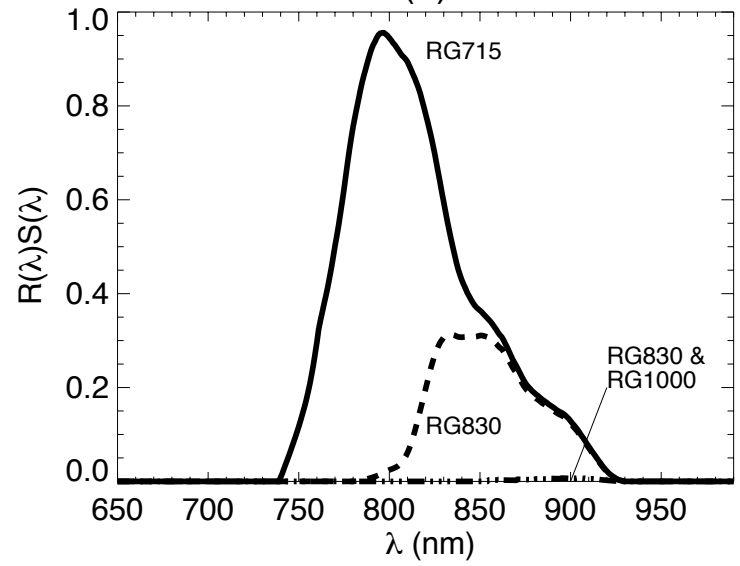

Figure 3. (a) Combined system response of the photo-detector including all optical components for various long pass filters (RG715, RG830, and a combination of RG830 \& RG1000) as compared to the actual FSRS spectrum. (b) Convoluted spectrum as seen by the photo-detector for various long-pass filters in arbitrary units. Since the actual shape of the spectrum is known the overall sensitivity of the detector including all optical components can be determined from the calibration at $527 \mathrm{~nm}$ to be $\mathrm{R}_{715}$ $=7.1 \times 10^{-5} \mathrm{~J} /(\mathrm{V} \mathrm{ns})$ with the RG715 filter, $\mathrm{R}_{830}=2.4 \times 10^{-4} \mathrm{~J} /(\mathrm{V} \mathrm{ns})$ with the RG830 filter, and $\mathrm{R}_{850+1000}=1.8 \times 10^{-2} \mathrm{~J} /(\mathrm{V}$ ns) using a combination of RG830 and RG1000.

\section{Forward Raman scattering measurements in large-scale length plasmas}

In experiments on gasbag targets $[9,5]$ at the Omega laser facility [4], we have measured considerable levels of FSRS from a high-intensity $\left(8 \times 10^{14} \mathrm{~W} / \mathrm{cm}^{2}\right)$ green $(527 \mathrm{~nm})$ interaction beam propagating through a mm-scale underdense plasma even in the presence of significant backward SRS.

The mm-size plasma was created by irradiating a $2.4 \mathrm{~mm}$ by $2.75 \mathrm{~mm}$ gasbag target [5] with 39 defocused laser beams at $351 \mathrm{~nm}$, delivering a total energy of $10.5 \mathrm{~kJ}$ in a 1 ns square pulse. The hydrocarbon gas fill at about 0.87 atm results in a plasma density around $5.5 \times 10^{20} \mathrm{~cm}^{-3}$, which corresponds to the current NIF hohlraum design for frequency doubled light $[10,11]$. A $1 \%$ argon dopand concentration was added as spectroscopic tracer. Some of the shots employed a CH-xenon mix with Xe-dopand concentrations between 3 and 18\% (by partial pressure). The plasma temperature was measured with Thomson scattering to be around $1.8 \mathrm{keV}$ as reported elsewhere [12]. These measurements confirmed the plasma parameters obtained in the radiationhydrodynamic simulations [13] that were used to model the backward SRS from these 
targets.[14] and form the basis for theoretical estimates of the SRSF gain obtained in this paper.

The $1 \mathrm{~ns}$ long frequency-doubled $(527 \mathrm{~nm})$ interaction beam had a variable energy between 20 and $360 \mathrm{~J}$ and was spatially smoothed with a distributed phase plate and by polarization smoothing [15], producing a $0.2 \mathrm{~mm}$ focal spot and an average intensity up to $8 \times 10^{14} \mathrm{~W} / \mathrm{cm}^{2}$. In some of the shots the beam was also temporally smoothed by spectral dispersion (SSD) by modulating the phase of the seed laser pulse at $1.1 \mathrm{~nm}$ bandwidth (at $1 \omega)[16]$.

Figure 4 shows streaked spectra of both forward and backward SRS recorded with the same spectrometer using two optical fibers with a $2.5 \mathrm{~ns}$ delay. Early in the $1 \mathrm{~ns}$ pulse the forward scattered light peaks at $820 \mathrm{~nm}$, while backward scattered light peaks at $860 \mathrm{~nm}$. This wavelength difference of about $40 \mathrm{~nm}$ is consistent with the local plasma parameters in the gasbag $\left(\mathrm{Te}=1.8 \mathrm{keV}, \mathrm{n}_{e} / \mathrm{n}_{c}=5.6 \%\right.$, where $\mathrm{n}_{c}$ is the critical density at $351 \mathrm{~nm}$ ) assuming that forward and backward scattered light originate from the same spatial location.

The backward SRS gain at early times shows peaks greater than 20 at two scattered light wavelengths: one at $860 \mathrm{~nm}$ from the scattering near the center of the bag and another at $870 \mathrm{~nm}$ on the density plateau just inside the blast wave. The phase velocity of the EPW that back scatters the laser light, $v_{p h} \sim 4.2 V_{t e}$, is small enough that the EPW is strongly damped (the spatial gain rate is less than the EPW damping rate) but weak enough that the gain is localized over a length of $0.7 \mathrm{~mm}$ or less than 4 speckle lengths. Thus, with the gain per speckle greater than one, strong backscatter is expected and measured. The SRSF light at $820 \mathrm{~nm}$ has the correct wavelength to be scattered at the center of the gasbag from a long wavelength EPW such that $\mathrm{k} \lambda_{\text {De }} \lesssim 0.05$ and $\omega_{\text {epw }} \simeq \omega_{p e}$ The phase velocity of the EPW for FSRS, $v_{p f} \sim 15 V_{t e}$, is so large that Landau damping is negligible. Only the weak collisional damping, $\nu_{l}=\nu_{e i} / 2$ remains where $\nu_{e i}$ is the electron-ion collision frequency. The SRSF gain is obtained from steady-state equations,

$$
\begin{aligned}
& \left(v_{g r} \frac{\partial}{\partial z}+\nu_{r}\right) a_{r}=\gamma_{0} a_{l} \\
& \left(v_{g l} \frac{\partial}{\partial z}+\nu_{l}+i \kappa(z)\right) a_{l}=\gamma_{0} a_{r},
\end{aligned}
$$

where $\gamma_{0}=\omega_{p e} k_{l}\left|V_{0}\right| /\left(4 \sqrt{\omega_{l} \omega_{r}}\right)$ is the growth rate for SRSF and $\kappa(z)=k_{0}-k_{r}-k_{l}$ is the phase mismatch between the laser light, the SRSF light, and the EPW. Here, $k_{0, r}=\sqrt{\omega_{0, r}^{2}-\omega_{p e}^{2}} / c$ and $k_{l}=\sqrt{\omega_{l}^{2}-\omega_{p e}^{2}} /\left(\sqrt{3} V_{t e}\right)$. The group velocities of the SRS light and the EPW satisfy $v_{g r} v_{g l}>0$ so only convective growth is possible. In the absence of spatial gradients, $\kappa=0$ and the scattered light and the EPW grow exponentially as, $a_{r, l} \simeq \exp \left(\kappa_{0} \mathrm{~L}\right)$ where the spatial growth rate, $\kappa_{0}=\gamma_{0} / \sqrt{v_{g r} v_{g l}}$, and $\mathrm{L}$ is the length of the uniform density. In fact the amount of SRSF is limited by spatial gradients in the gasbag. The biggest growth occurs at the center of the bag at a local minimum of the density where the local density gradient, $d N_{e} / d z=0$ but $d^{2} N_{e} / d z^{2}>0$. The theory for the linear gain coefficient has been obtained [17] in the weak, $\nu_{l} / v_{g l}<\left|d \ln a_{l} / d z\right|$ 
and strong EPW damping limits, $\nu_{l} / v_{g l}>\left|d \ln a_{l} / d z\right|$. Following reference [17], we take $\kappa(z)=\frac{1}{2} \kappa^{\prime \prime} \mathrm{z}^{2}$ at the presumed matching point at $\mathrm{z}=0$, solve Eq. (2) in the weak damping limit with the WKB approximation,

$$
\begin{aligned}
& \Psi^{\prime \prime}+\mathrm{Q}(\mathrm{z}) \Psi=0 \\
& \mathrm{Q}(\mathrm{z}) \quad=\left(\frac{\left(\kappa^{\prime \prime}\right)^{2} \mathrm{z}^{4}}{4}-\kappa_{0}^{2}\right)
\end{aligned}
$$

where $\Psi=a_{r} \exp \left(-\frac{i}{2} \int d z \kappa(z)\right)$ such that $\Psi \simeq \exp \left(\int \sqrt{Q(z)} d z\right)$, and find the energy gain is

$$
\mathrm{G}_{\kappa^{\prime \prime}}=2 \log \Psi=7 \frac{\gamma_{0}^{3 / 2}}{\left(\sqrt{\kappa^{\prime \prime}}\left(v_{g r} v_{g l}\right)^{3 / 4}\right)} .
$$

In the limit of strong damping, we can omit the $d a_{l} / d z$ term to find

$$
\frac{\mathrm{d}}{\mathrm{dz}} a_{r}=\left(\frac{\kappa_{0}^{2}}{\frac{\nu_{l}}{v_{g l}}+i \frac{\kappa^{\prime \prime} z^{2}}{2}}\right) a_{r}
$$

and the energy gain,

$$
\mathrm{G}_{\nu \kappa^{\prime \prime}}=2 \pi \frac{\gamma_{0}^{2}}{\left(\mathrm{v}_{\mathrm{gr}} \sqrt{\kappa^{\prime \prime} \nu_{\mathrm{l}} \mathrm{v}_{\mathrm{gl}}}\right)} .
$$

In a given physical case, the smaller of these two gains applies. At the matching surface, $\kappa^{\prime \prime} \simeq \omega_{p e}^{2} /\left(\omega_{l} v_{g l} L_{2}^{2}\right)$ where $L_{2}^{-2}=N_{e}^{-1} d^{2} N_{e} / d z^{2}$. We can find the effective length over which the SRSF growth occurs by comparing $2 \kappa_{0}$ with $\mathrm{G}_{\kappa^{\prime \prime}}$ with the result that $\mathrm{L}_{\text {eff }} \sim 3.5 \sqrt{\kappa_{0} / \kappa^{\prime \prime}} \sim 3.5 \sqrt{\gamma_{0} / \omega_{\mathrm{pe}}}\left(\mathrm{v}_{\mathrm{gl}} / \mathrm{v}_{\mathrm{gr}}\right)^{1 / 4} \mathrm{~L}_{2} \sim 0.04 \mathrm{~L}_{2}$ where the numerical value was obtained for a laser intensity of $1 \times 10^{14} \mathrm{~W} / \mathrm{cm}^{2}$ and an electron density of $N_{e}=5 \times 10^{20} \mathrm{~cm}^{-3}$.

From the HYDRA simulations of this plasma [13] we find at 800 ps after the $351 \mathrm{~nm}$ laser heater pulse is incident on the gasbag (about 300 ps into the $527 \mathrm{~nm}$ interaction pulse) that the density scalelength, $\mathrm{L}_{2}=0.14 \mathrm{~cm}$, and the gains $\mathrm{G}_{\kappa^{\prime \prime}}=5$ and $\mathrm{G}_{\nu \kappa^{\prime \prime}}=3$ for an average laser intensity of $1 \times 10^{14} \mathrm{~W} / \mathrm{cm}^{2}$. The growth occurs over a small region, $\mathrm{L}_{\mathrm{eff}} \sim 60 \mu \mathrm{m}$ that is not resolved by hydrodynamic simulations. A gain of 3 is not sufficient for the level of SRSF measured in the experiments but, for an average laser intensity of $4 \times 10^{14} \mathrm{~W} / \mathrm{cm}^{2}$ at the center of the gasbag, $\mathrm{G}_{\kappa^{\prime \prime}} \sim \mathrm{G}_{\nu \kappa^{\prime \prime}}=13$ which probably is sufficient especially if the laser beam speckles and filamentation are taken into account. Speckle enhancement of the growth is particularly effective when the entire gain occurs over a length less than the speckle length, $\sim 200 \mu \mathrm{m}$ in this case. Note, that the backward SRS gain for an incident intensity of $4 \times 10^{14} \mathrm{~W} / \mathrm{cm}^{2}$ or $2 \times 10^{14} \mathrm{~W} / \mathrm{cm}^{2}$ at the center of the bag is 80 so much more backward than forward SRS is expected.

Without the loss to SRS backscatter, we estimate from transmission measurements that $60 \%$ of the incident light will reach the middle of the gasbag to drive FSRS. With the ratio of SRS frequency to incident light frequency of 1.6, we estimate that $60 \%$ of the SRS light will be transmitted through the blast wave. Given that the measured backscattered SRS is about $10 \%$ at and above $4 \times 10^{14} \mathrm{~W} / \mathrm{cm}^{2}$, the actual loss to the 
incident light is closer to $30 \%$ when the energy taken by the EPW is accounted for. Thus, about $1 / 2$ of the light that reaches the middle of the bag is SRS backscattered which leaves $30 \%$ of the incident light to drive FSRS.

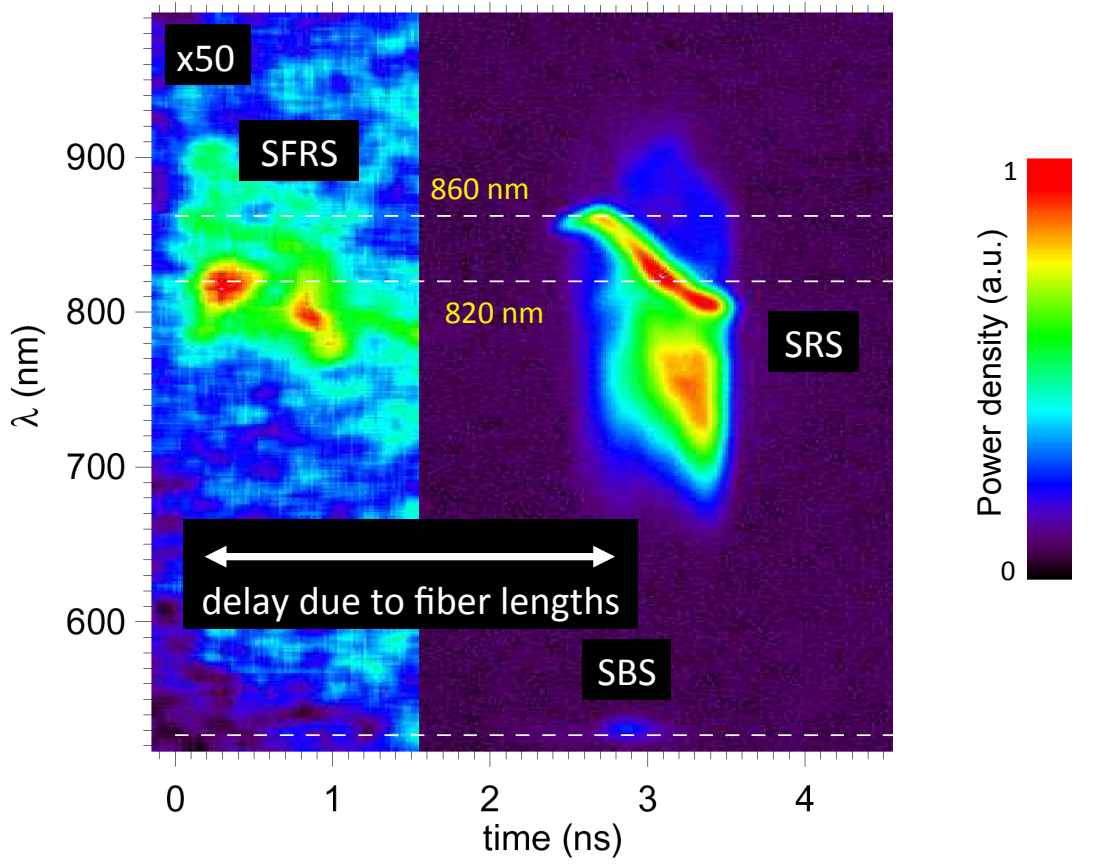

Figure 4. Streaked spectrum of forward (FSRS) and backward (SRS) Raman scattered light at $8 \times 10^{14} \mathrm{~W} / \mathrm{cm}^{2}$ Both spectra were combined on the same streaked spectrometer using optical fibers with different length and thus a relative delay of 2.5 ns between SRS and FSRS. The first 1.5 ns of the data were scaled up in intensity by a factor of 50 to make the FSRS signal visible which is much smaller than the SRS signal. At early times the FSRS signal peaks at $820 \mathrm{~nm}$, while the SRS signal peaks at $860 \mathrm{~nm}$

In these experiments the maximum observed FSRS level was of the order of 0.1 $\%$ of the total incident light, with a measured SRS level of $8 \%$. However, beam transmission measurements suggest that a significant fraction $(\sim 80-90 \%)$ of both the up-shifted forward and backward scattered light was absorbed in the high-density blastwave originating from the polyimide skin of the gasbag [5, 12]. The actual level of FSRS light inside the underdense plasma was therefore significantly higher, and close to $0.5-1 \%$, while the interaction beam intensity driving FSRS was significantly lower $\left(\sim 4 \times 10^{14} \mathrm{~W} / \mathrm{cm}^{2}\right)$. Figures 5 and 6 show the variation of the forward scattered energy with laser intensity and plasma density. We observe a nonlinear increase of FSRS with beam intensity. The forward scattered light does not depend much on dopandconcentration or density but decreases slightly with SSD. Plotting the ratio between forward scattered light and unshifted transmitted light at the laser line $(527 \mathrm{~nm})$ reveals a stronger dependence on density and SSD. 
(a)

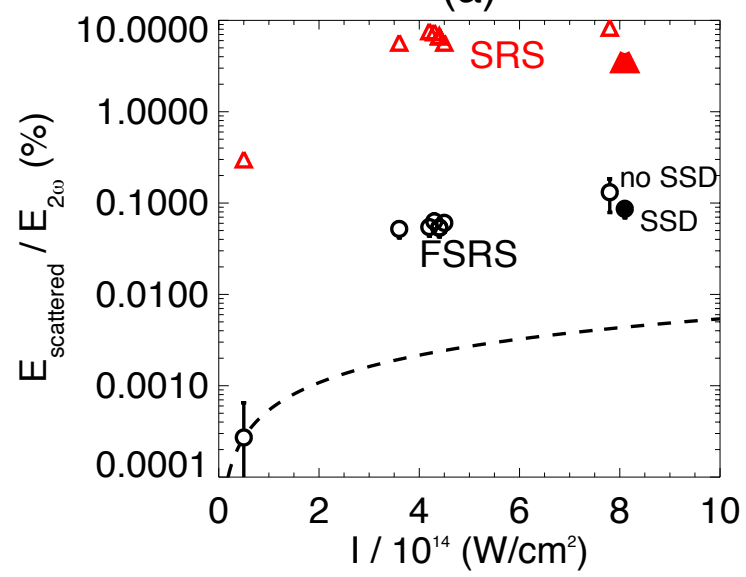

(b)

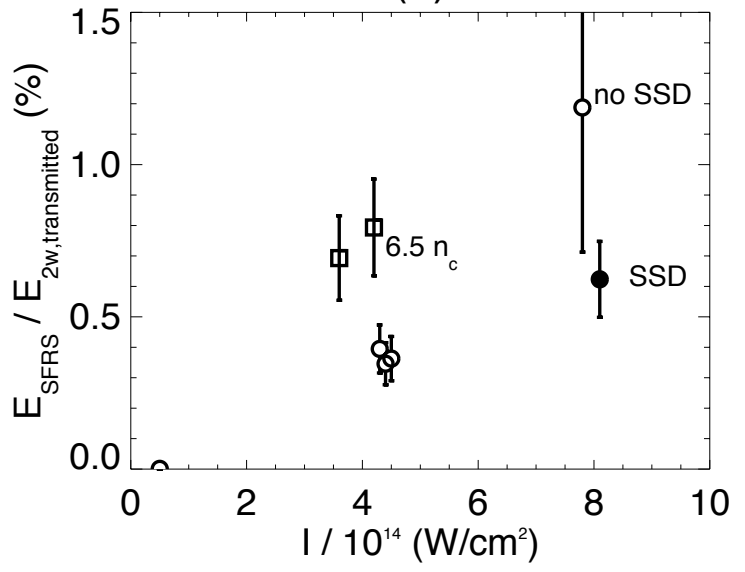

Figure 5. (a) Fraction of forward scattered energy of the total interaction beam energy versus beam intensity. We observe a nonlinear increase of FSRS with beam intensity. The dashed line indicates a linear fit through the origin and the lowest intensity point at $0.5 \times 10^{14} \mathrm{~W} / \mathrm{cm}^{2}$. The five data points around $4 \times 10^{14} \mathrm{~W} / \mathrm{cm}^{2}$ had varying Xe-concentrations between 0 and $18 \%$ and varying density between 5 and 6.5 $\mathrm{n}_{c}$ but similar forward scattering fractions around $0.1 \%$. The two high intensity shots $\left(8 \times 10^{14} \mathrm{~W} / \mathrm{cm}^{2}\right.$ were performed with $1 \%$ argon dopand. Turning on SSD reduces the FSRS fraction from $0.13 \%$ to $0.08 \%$. The measured backward Raman scattered energy is shown for comparison and follows the same trend but is about a factor of 80 higher than the forward scattering level. (b) Ratio between forward scattered light and transmitted light at the laser line $(527 \mathrm{~nm})$ versus beam intensity. The two shots at $4 \times 10^{14} \mathrm{~W} / \mathrm{cm}^{2}$ that have a higher FSRS energy had slightly larger plasma densities $\left(\mathrm{n} / \mathrm{n}_{c}=6.5 \%\right)$.

(a)

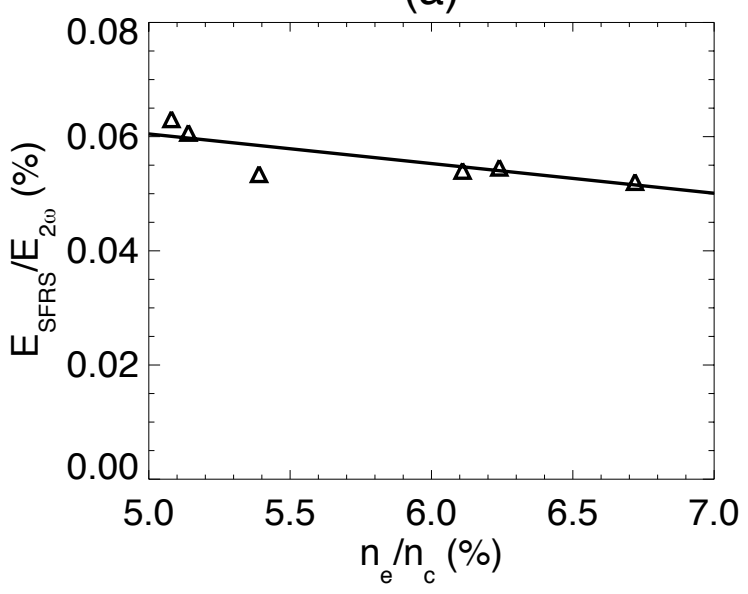

(b)

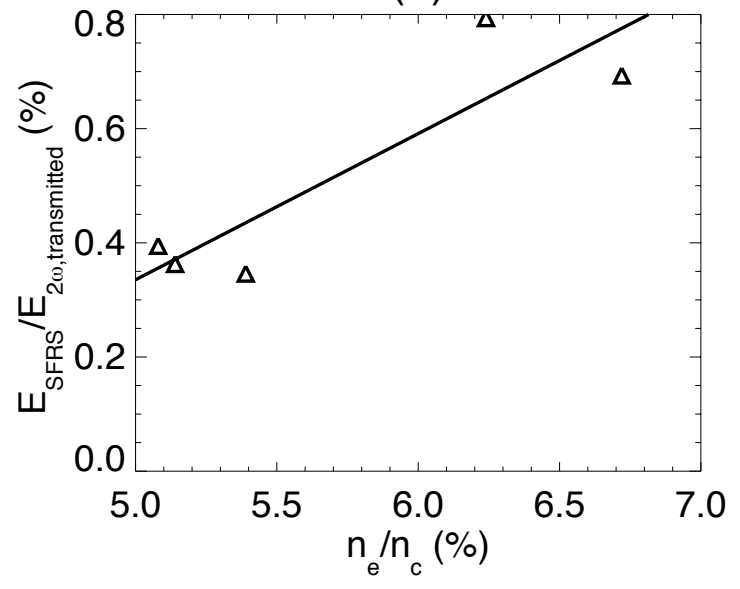

Figure 6. (a) Fraction of forward scattered energy of the total incident beam energy versus plasma density (in critical density at $351 \mathrm{~nm}$ ) for similar target parameters and laser energies $(\sim 150 \mathrm{~J})$. (b) Ratio between forward scattered and unshifted transmitted light versus density. While the measured FSRS level slightly decreases with increasing density the ratio between forward scattered light and unshifted light increases. 
Figure 7 shows forward scattering signals in arbitrary units recorded with different long-pass filters but otherwise identical laser-target parameters (i.e. $\mathrm{E}_{2 \omega}=150 \mathrm{~J}$, $\mathrm{n} / \mathrm{n}_{c}=6 \%, 1 \%$ argon dopand concentration, no SSD) and thus FSRS levels. The raw signal decreases strongly with increasing cut-off wavelength of the filter since less of the FSRS spectrum is transmitted to the detector. Applying the appropriate calibration factor for each filter (Fig. 3) recovers a detector independent FSRS signal, which is identical for all shot within the error bars (red circles). This is an independent validation that the FSRS spectrum peaks around $800 \mathrm{~nm}$ and that the FSRS energy sensor measures only forward scattered light.

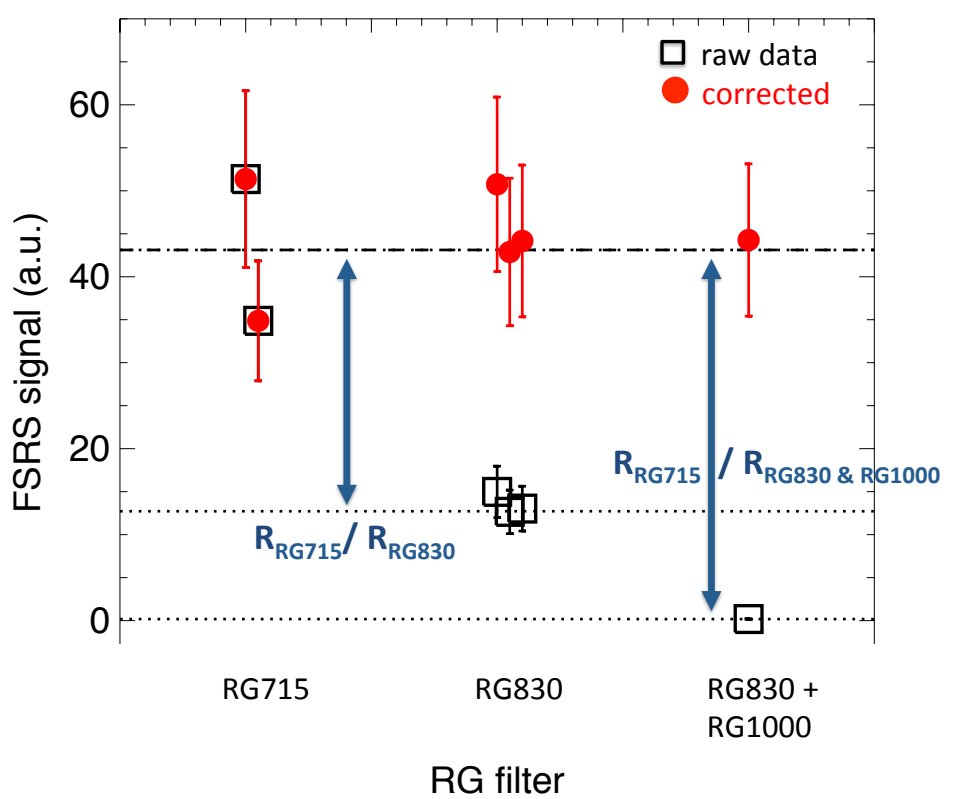

Figure 7. FSRS signal (in arbitrary units) for various long-pass filters but identical laser-target parameters $\left(\mathrm{E}_{2 \omega}=150 \mathrm{~J}\right)$. The black rectangles show the raw data, which decreases strongly with cut-off wavelength of the filter. By compensating for the spectral response of the system, i.e. multiplying the $R G 830$ raw data with $R_{830} / R_{750}$ $=3.4$, and the RG1000 data with $\mathrm{R}_{830+1000} / \mathrm{R}_{750}=254$ the same relative FSRS level is reconstructed (red circles), which is an independent validation of the FSRS spectrum and confirms that the photo-multiplier measures only FSRS light.

\section{Conclusion}

Using a spectrally and temporally resolved transmitted beam diagnostics we have measured forward stimulated Raman scattering from a hot long-scale-length laser produced plasma. The ratio between backward and forward scattered light is of the order of 80 with a measured level of forward scattered light as high as $0.1 \%$ of the probe beam energy. However, beam transmission measurements reported elsewhere [5] suggest 
that a significant fraction $(\sim 80-90 \%)$ of the up-shifted forward- and backward scattered light was absorbed in the high-density blast-wave originating from the gas-bag skin. The actual level of FSRS inside the under dense plasma was therefore significantly higher, and closer to $0.5-1 \%$. The shift of the peak wavelengths of FSRS and SRS of around $40 \mathrm{~nm}$ is consistent with the local plasma parameters. The results are of interest to laser-light coupling to ignition hohlraums at the NIF. The plasma-scales at NIF are 3-5 times larger but the FSRS growth rates are similar. While backward SRS is inhibited in NIF hohlraums by the strong Landau damping when $\mathrm{k} \lambda_{D}=0.3$ and possibly a loss of resonance when $\mathrm{k} \lambda_{D}=0.53$, forward $\operatorname{SRS}\left(\mathrm{k} \lambda_{D}=0.1\right)$ is practically not damped. The ratio between FSRS and SRS levels are therefore expected to be higher at NIF than in the experiments described here. Significant levels of backward SRS are typically observed in NIF hohlraum experiments [2] but there is currently no measurement of forward SRS at NIF.

This work performed under the auspices of the U.S. Department of Energy by Lawrence Livermore National Laboratory under Contract DE-AC52-07NA27344.

\section{References}

[1] Lindl J, Amendt P, Berger R.L, Gail Glendinning S, Glenzer S.H, Haan S.W, Kauffman R.L, Landen O.L, Suter L.J 1995 Phys. Plasmas 2, 3933

[2] Glenzer S.H et al. 2011 Phys. Rev. Lett. 106, 085004

[3] Turner R, Estabrook K, Drake R, Williams E, Kornblum H, Kruer W, Campbell E 1986 Phys. Rev. Lett. $\mathbf{5 7}(14), 1725$

[4] Soures J 1991 J. Fusion Energy 10(4), 295

[5] Niemann C, Divol L, Froula D, Gregori G, Jones O, Kirkwood R, MacKinnon A, Meezan N, Moody J, Sorce C, Suter L, Bahr R, Seka W, Glenzer S 2005 Phys. Rev. Lett. 94, 085005

[6] Mackinnon A, Shiromizu S, Antonini G, Auerbach J, Haney K, Froula D, Moody J, Gregori G, Constantin C, Sorce C, Divol L, Griffith R, Glenzer S, Satariano J, Whitman P, Locke S, Miller E, Huff R, Thorp K, Armstrong W, Bahr R, Seka W, Pien G, Mathers J, Morse S, Loucks S, Stagnitto S, 2004 Rev. Sci. Instrum, 75, 3906

[7] Niemann C, Antonini G, Compton S, Glenzer S, Hargrove D, Moody J, Kirkwood R, Rekow V, Satariano J, Sorce C, Armstrong W, Bahr R, Keck R, Pien G, Seka W, Thorp K 2004 Rev. Sci. Instrum. 75(10), 4171

[8] Hamamatsu Photonics, 2004 internal communication

[9] MacGowan, B.J, Afeyan B., Back C., Berger R, Bonnaud G, Casanova M, Cohen B, Desenne D, DuBois D, Dulieu A, Estabrook K, Fernandez J, Glenzer S, Hinkel D, Kaiser T, Kalantar D, Kauffman R, Kirkwood R, Kruer W, Langdon A, Lasinski B, Montgomery D, Moody J, Munro D, Powers L, Rose H, Rousseaux C, Turner R, Wilde B, Wilks S, Williams 1996 Phys. Plasmas 3, 2029

[10] Suter, L.J, Glenzer S, Haan S, Hmmel B, Manes K, Meezan N, Moody J, Spaeth M, Divol L, Oades K, Stevenson M 2004 Phys. Plasmas 11, 2738

[11] Stevenson R.M, Suter L., Oades K., Kruer W., Slark G., Fournier K., Meezan N., Kauffman R., Miller M. Glenzer S., Niemann C., Grun J.,Davis J., Back C., Thomas B. 2004 Phys. Plasmas 11, 2709

[12] Niemann C, Berger R.L, Divol L, Froula D, Jones O, Kirkwood R, Meezan N, Moody J, Ross J, Sorce C., Suter L, Glenzer S. 2008 Phys. Rev. Lett. 100, 045002 
[13] Meezan N.B, Berger R.L, Divol L, Froula D.H, Hinkel D.E, Jones O.S, London R.A, Moddy J.D, Marinak M.M, Niemann C, Neumeyer P.B, Prisbrey S.T, Ross J.S, Williams E.A, Glenzer S.H, Suter L.J, Phys. Plasmas 14, 056304 (2007)

[14] Moody J.D, Divol L, Froula D.H, Glenzer S.H, Gregori G, Kirkwood R.K, Mackinnon A, Meezan N, Niemann C, Suter L.J, Bahr R, Seka W, Phys. Plasmas 16, 062704 (2009)

[15] Monro D, Dixit S, Langdon B, Murrya J 2004 Applied Optics 43(36), 6639

[16] Skupsky S, Short R, Kessler T, Craxton R, Letzring S, Soures J 1989 J. Appl. Phys. 66, 3456

[17] E. A. Williams, LLNL ATR 1986 